\title{
Congruence of Coherence Peaks of Daily Geomagnetic Activity and Total Earthquakes with Mintaka's (Orion's Belt) 5.7 Day Periodicity: Potential Implications for Astrobiology
}

\author{
Blake T. Dotta, Joseph M. Caswell and Michael A. Persinger* \\ Laurentian University, Sudbury, Ontario, Canada \\ "Corresponding author: Michael A. Persinger, Professor, Quantum Biophysics Laboratory, Biomolecular Sciences and Behavioural Neuroscience Programs, \\ Laurentian University, Sudbury, Ontario, Canada P3E 2C6, Tel: 705675 1151; E-mail: mpersinger@laurentian.ca
}

Received date: Jul 22, 2014; Accepted date: Oct 9, 2014; Published date: Oct 13, 2014

Copyright: (c) 2014 Dotta BT, et al. This is an open-access article distributed under the terms of the Creative Commons Attribution License, which permits unrestricted use, distribution, and reproduction in any medium, provided the original author and source are credited

\begin{abstract}
A single strong frequency-dependence was revealed by cross-spectral analysis between daily geomagnetic activity and total numbers of daily earthquakes between 2009 through 2013 that exhibited a 5.7 day cycle. We examined the potential for association with Mintaka's double star orbital periodicity of 5.73 days. Calculations of gravitational force and equivalent energies between the earth and Mintaka as well as the variations with orbital periodicity indicated reliable radiant flux densities upon the terrestrial surface in the order of $10^{-11} \mathrm{~W} \cdot \mathrm{m}^{-2}$. This is within the same order of magnitude as background photon emissions whose increase occurs conspicuously before major earthquakes and are related to geomagnetic activity. This magnitude of power has recently been measured from preparations of brain tissue and from human cerebrums while subjects sat in hyper-dark settings and engaged in imagination. The power density also matches the gravitational energy within a human brain mass from empirical measurements of the variation in $\mathrm{G}$. The convergence of quantitative solutions suggests that shared periodicities from some very distant stellar objects may affect terrestrial processes assuming the power densities are congruent.
\end{abstract}

Keywords: Mintaka double star; 5.7 Day periodicity; Geomagnetic activity; Seismic activity; $10^{-11} \mathrm{~W} \cdot \mathrm{M}^{-2}$; Biological activity; Physical cosmobiology

\section{Introduction}

Although the effects from background gravitational components upon biological processes have been considered negligible, recent measurements within the expanding discipline of quantum biology have suggested that very small energies are associated with important processes. For example the energy required to sequester one base nucleotide to a sequence of RNA is about $10^{-20}$ Joules $(\mathrm{J})$ and occurs in about $1 \mathrm{~ms}$ [1]. Similarly the energy associated with the $1 \mathrm{~ms}$ duration change in voltage of the plasma membrane of the axon upon a unit charge is about $10^{-20} \mathrm{~J}$ [1]. The energy associated with the application of the gravitational force (about $10^{-12} \mathrm{~N}$ ) of the earth upon a standard mammalian cell with a mass of about $10^{-13} \mathrm{~kg}$ across the plasma membrane $\left(10^{-8} \mathrm{~m}\right)$ is $\sim 10^{-20} \mathrm{~J}$ [2]. There is both quantitative and empirical evidence that even subtle lunar tidal forces affect cells and are associated with differential emissions of photons [3] that are synchronized over the terrestrial surface.

The current estimates of the average density of energy within the universe are around $0.3 \cdot 10^{-9} \mathrm{~J} \cdot \mathrm{m}^{-3}$ [4]. When distributed over the distance of the neutral hydrogen line $(21 \mathrm{~cm})$, the power density per second would be in the order of $10^{-11} \mathrm{~W} \cdot \mathrm{m}^{-2}$. The radiant flux densities are within the range measured for photons emitted from the earth which increase a few days before very large earthquakes [5]. This value is also within the same order of magnitude as that measured from the cerebrums of human subjects sitting in very dark environments and engaging in imagination compared to more mundane cognitive activities [6]. Assuming $10^{-20} \mathrm{~J}$ per action potential and a cross sectional area of the cerebral cortices of $10^{-2} \mathrm{~m}^{2}, \sim 10^{-11} \mathrm{~W} \cdot \mathrm{m}^{-2}$ of photon flux during the biophysical activities associated with thinking would be associated with $\sim 10^{6}$ to $10^{7}$ neurons discharging within the range of about $10 \mathrm{~Hz}$, the major peak power density of cerebral activity. Considering that the activity of only one neuron can change the state of the entire cerebral cortices [7] and affect behavior [8], such subtle energies may be more important than expected.

The influence from the mass $\left(\sim 2 \cdot 10^{30} \mathrm{~kg}\right)$ of the sun upon terrestrial organisms would be expected because of its proximity. Traditionally the gravitational effects upon terrestrial biological systems by more distant stellar masses have been considered trivial. During our explorations of the quantitative coupling between fluctuations in daily geomagnetic activity and the total numbers of global seismic events we isolated a cross spectral coherence of these two processes of $2.03 \cdot 10^{-6}$ $\mathrm{Hz}$ or approximately 5.7 days. Because of our interest in the three stars of Orion's Belt (particularly the triple system $\delta$ Ori-A, $-\mathrm{B}$, and $-\mathrm{C}$ ) from more traditional astronomical perspectives we recognized the similarity to the 5.73 day rotational time of the double star Mintaka. Although likely to be coincidence we reasoned that such "accidental" concurrence should be at least examined from a quantitative perspective. Here we present evidence that the association may not be coincidence and may reflect some general common process shared by stellar systems that is relevant to astrobiology.

\section{Geomagnetic and Global Seismic Energy Coherence (5.7 days)}

There has been a long history of discerning quantitative relationships between changes in geomagnetic activity, the release of seismic energy on a global scale, and relatively small variations in solar activity as inferred by Solar Flux Units centralized at $2.8 \mathrm{GHz}$. The lag/ lead relationships involving daily $\Delta t$, versus the over inclusive monthly or yearly increments of analyses, suggest there is a third 
Citation: Dotta BT, Caswell JM, Pesinger MA (2014) Congruence of Coherence Peaks of Daily Geomagnetic Activity and Total Earthquakes with Mintaka's (Orion's Belt) 5.7 Day Periodicity: Potential Implications for Astrobiology. JAstrobiolOutreach 2: 120. doi: $10.41721 / 2332-2519.1000120$

Page 2 of 4

factor to which perturbations in solar activity, geomagnetic activity and earthquakes are related. A potentially cosmological and quantumbased explanation for these intercorrelations has been developed by Vares and Persinger [9]. In the present analyses daily aa indices (nT) from 2009 to 2013 (number of days=1826) were acquired from the International Service for Geomagnetic Indices (ISGI; isgi.latmos.ipsl.fr/). The daily total numbers of earthquakes $>0.01 \mathrm{M}$ recorded by the United States Geological Survey (USGS; www.usgs.gov/) were also obtained. All statistical procedures were conducted using SPSS 17 software.

The aa index values were de-trended using a linear regression with time as the independent variable and employing the residual values for subsequent analyses. Cross-spectral measures were used in order to evaluate potential frequency-dependent coherence between daily geomagnetic (de-trended aa index) and earthquake activity and the associated phase lag/lead relationships. By obtaining frequencydomain data from two variables with independent spectral analyses, various cross-spectral characteristics can be derived to produce appropriate statistics to examine potential frequency-dependent causal relationships between signals.

The primary measure of interest for the current analysis was the cross-spectral coherence. Derived from the independent or autospectral densities of each variable and the cross-spectral density (Fourier transform of a cross-correlation function), this value represents a frequency-domain analogue to a standard correlation coefficient and can be interpreted as such (e.g., proportion of shared variance between variables within a specific frequency). This method can be used to estimate the power transfer between two signals $(x[t]$ and $y[t])$, and is computed using the formula $C_{x y}=\left|S_{x y}\right|^{2} /\left(S_{x x} \cdot S_{y y}\right)$, where $S_{x y}=$ cross-spectral density between variables, $S_{x x}$ and $S_{y y}=$ independent spectral densities of $x[t]$ and $y[t]$, and $\left|S_{x y}\right|=$ magnitude of spectral density. Final values used for evaluation represent the squared coherence between variables $\left(\mathrm{CH}^{2}\right)$.

Multiple Fourier transforms were applied to geomagnetic (aa index) and earthquake values using varied window sizes (Tukey-Hamming) to better isolate consistent squared coherence peaks of $\mathrm{CH}^{2}>0.80$. Cross-spectral analyses were conducted and a total of $n=913$ standardized frequencies were extracted. True frequency values were computed using the spectral unit frequencies $\left(\mathrm{f}_{\mathrm{i}}\right)$ with $\left(1 / \mathrm{f}_{\mathrm{i}}\right) \cdot 86400 \mathrm{~s}=$ $\mathrm{f}_{\mathrm{s}}, 1 / \mathrm{f}_{\mathrm{s}}=\mathrm{Hz}$.

A single distinct coherence peak $>0.80$ between signals was identified across window sizes (Figure 1) at a frequency of $2.03 \cdot 10^{-6}$ $\mathrm{Hz}$, indicating a strong frequency-dependent relationship between daily aa indices and total numbers of recorded earthquakes for a specific cycle of $\sim 5.702$ days.

The phase spectrum was then assessed for lag/lead effects between signals at the previously identified frequency. A positive phase of 0.987 radians was revealed and divided by $2 \pi$ to obtain the difference in proportions of a cycle. This value was shown to be relatively small $\left(0.157=\sim 16 \%\right.$ of a full 5.702 day cycle), suggesting that the $2.03 \cdot 10^{-6}$ $\mathrm{Hz}$ geomagnetic cycle lead the earthquake cycle by approximately 0.912 of a single day or 21.88 hours. While both variables demonstrate marked coherence within this specific frequency, the phase relationship may be further indicative of the geomagnetic field as a potential source of variance in the context of daily numbers of earthquakes or that both share variance with a third factor but exhibit differential response latencies.

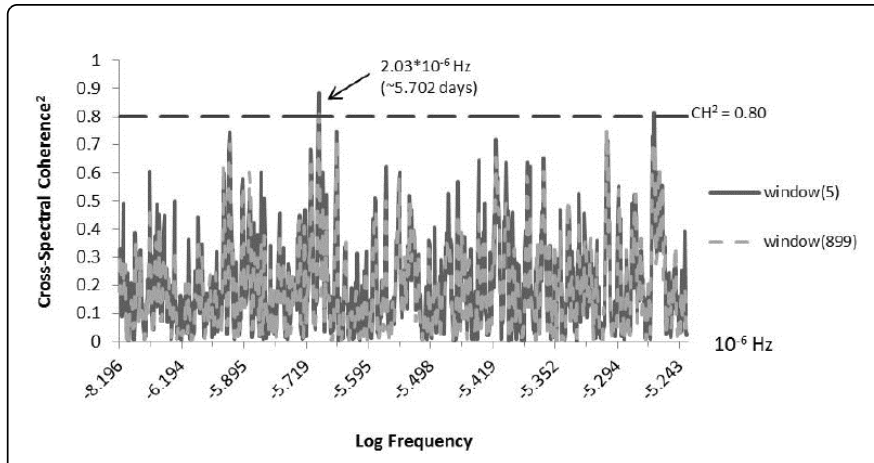

Figure 1: Cross-spectral coherence for two extreme Fourier window sizes (Tukey-Hamming); arrow indicates distinct consistent $\mathrm{CH}^{2}$ peak at $2.03 \cdot 10^{-6} \mathrm{~Hz}$ that was consistent across window sizes. Additional peak $\mathrm{CH}^{2} \sim 0.80$ was consistent across various FFT windows.

\section{Photon Flux Density Equivalence and Characteristics of Mintaka (Orion's Belt)}

According to classical bright star catalogues, e.g., the Smithsonian Astrophysical Observatory Star Catalogue, the double star system labeled visually as Mintaka is $\sim 6.5 \cdot 10^{18} \mathrm{~m}$ (690 LY) from earth. Delta Orion ( $\delta$ Orionis) contains class $\mathrm{B}$ and $\mathrm{O}$ stars. Mintaka $\mathrm{A}$ is composed of three stars; one rotates around the other two very proximal stars every 5.73 days. Depending upon source data, each of the stars are $4 \cdot 10^{31} \mathrm{~kg}$ (20 solar masses). The separation between the two stars is inferred by spectral anomalies to be in the order of $0.3 \mathrm{arc}$ seconds. The inference of mutual orbiting stars is also based upon the transient diminishment of $0.2 \mathrm{M}$ (magnitude) relative to the reference that corresponds with the orbital period.

The radiant flux density of Mintaka (Apparent Magnitude=2.23) can be estimated by referencing the known relationship between Sirius, with an apparent brightness of $-1.46 \mathrm{M}$ which is $\sim 10^{-7} \mathrm{~W} \cdot \mathrm{m}^{-2}$. The difference for this logarithmic relationship is 3.69 , such that $\log 10$ of $10^{-10.69}$ is $\sim 10^{-11} \mathrm{~W} \cdot \mathrm{m}^{-2}$ at earth distance. The $0.2 \mathrm{M}$ fluctuation attributed to orbital eclipsing would primarily affect the coefficient but not the order of magnitude of this radiant flux density.

This value is within the range of magnitude of the power densities of photons that are emitted from the right hemisphere of human volunteers sitting in hyper-dark conditions while engaging in imagination or activated cognition but not while "thinking" about mundane events [6]. It is relevant that that solution congruent with biological function occurs for the apparent magnitude of Mintaka, rather than the absolute magnitude. In other words the power density of the light reaching earth rather than its values adjusted for distance is most convergent with biological levels of photon emissions.

\section{Gravitational Forces and Energies}

From traditional application of Newton's gravitational equation which is based upon the product of the masses of the objects being considered and $\mathrm{G}\left(6.67 \cdot 10^{-11} \mathrm{~m}^{3} \cdot \mathrm{kg}^{-1} \mathrm{~s}^{-2}\right)$ divided by the square of the distance, the force between the primary stars (each with a mass of 20 suns) associated with the 5.73 day periodicity and earth would be the product of $8 \cdot 10^{31} \mathrm{~kg}$ and $6 \cdot 10^{24} \mathrm{~kg}$ (earth's mass) divided by $4.25 \cdot 10^{37}$ $\mathrm{m}^{2}$ and when multiplied by $\mathrm{G}$, is, $7.52 \cdot 10^{8} \mathrm{~N}$. The intrinsic fluctuation 
Citation: Dotta BT, Caswell JM, Pesinger MA (2014) Congruence of Coherence Peaks of Daily Geomagnetic Activity and Total Earthquakes with Mintaka's (Orion's Belt) 5.7 Day Periodicity: Potential Implications for Astrobiology. JAstrobiolOutreach 2: 120. doi: $10.41721 / 2332-2519.1000120$

Page 3 of 4

per second for the 5.73 days $\left(4.95 \cdot 10^{5} \mathrm{~s}\right)$ would be $1.52 \cdot 10^{3} \mathrm{~N} \cdot \mathrm{s}^{-1}$. If distributed across the $21 \mathrm{~cm}$ neutral hydrogen line, which appears to be a fundamental temporal coupling at both Planck's spaces [10] and biological functions [11], the power would be $3 \cdot 2 \cdot 10^{2} \mathrm{~W}$.

This value becomes relevant in an astrobiological context when it is distributed over the surface of the earth $\left(5.1 \cdot 10^{14} \mathrm{~m}^{2}\right)$. The radiant power density would be, on average, $0.6 \cdot 10^{-12} \mathrm{~W} \cdot \mathrm{m}^{-2}$. This value is within the background fluctuation levels of photon emissions from the earth, at least in our area [12], as measured by photomultplier units. We [5] have found that background photon emissions increased by a factor of about 100 for several days before the Japanese 9.0 M seismic event and the Chilean 8.8 M seismic event. Smaller increases in background photon emissions have been routinely associated with seismic events as low as $6.8 \mathrm{M}$.

Recent increases in the sensitivity of global networks for the recording of seismic activity have allowed more accurate estimates of the total energy released within various increments of magnitudes. During the same interval (1,826 days) we employed to discern the 5.7 day coherence in geomagnetic activity and total numbers of daily earthquakes, the total energy per day from 0.01 to $1 \mathrm{M}$ seismic events was about $7.56(\mathrm{SD}=2.28) \cdot 10^{7} \mathrm{~J}$ or about $0.88 \mathrm{~J} \cdot \mathrm{s}^{-1}$. This is an important fact because $38 \%$ of all of the earthquakes during the five years of measurement were within that category. In fact $73 \%$ of the numbers of earthquakes occurred within the M 0.01 to 2 range. Hence a substantial proportion of the variance associated with the total numbers of daily earthquakes that were coupled within an intrinsic fluctuation of 5.7 days with geomagnetic activity involved the very weakest events.

When the value of $0.88 \mathrm{~J} \cdot \mathrm{s}^{-1}$ was distributed over the surface area of the earth $\left(5.1 \cdot 10^{14} \mathrm{~m}^{2}\right)$ the power density is about $0.2 \cdot 10^{-11} \mathrm{~W} \cdot \mathrm{m}^{-2}$. This is the same order of magnitude and almost the same coefficient as the power density associated with the potential orbital changes over the 5.7 day interval displayed by the double star system in Mintaka. Although the time-lagged coupling between very low magnitude seismic events and later larger intensity seismic events is not always present, this frequent sequence may suggest that some zeitgeber might occasionally interact given optimal conditions.

The temporal associations between fluctuations in photon emissions and geomagnetic activity have been quantified in our laboratory [12]. In general increases in photon emission flux densities are associated with decreases in geomagnetic activity. The average slope indicates that for every $1 \mathrm{nT}$ increase in geomagnetic fluctuations there is a $\sim 10^{-12} \mathrm{~W} \cdot \mathrm{m}^{-2}$ decrease in photon emissions. Changes within the 1 to $20 \mathrm{nT}$ range for the solar wind as it interacts with the magnetopause have been correlated negatively in two separate measurements [13] with the variations in $G$ [14], which are in the order of $10^{-3}$ of the actual constant. All of these empirical measures suggest that very small energies associated with geomagnetic activity, the background fluctuations in $\mathrm{G}$, and global seismic energy radiation or some process that determines the numbers of discrete seismic events per day may share a source of variance with which more distal sources could interact.

\section{Mintaka-Earth Gravitational Energies and Cerebral Activities}

The flux densities of $\sim 10^{-12}$ and $\sim 10^{-11} \mathrm{~W} \cdot \mathrm{m}^{-2}$ are also the same magnitudes associated with photon emissions from the right hemispheres of human subjects sitting in very dark rooms engaging in imagination rather than mundane cognitive tasks. Assuming approximately $10^{-20} \mathrm{~J}$ per action potential and a cross sectional area of the human cerebrum to be about $10^{-2} \mathrm{~m}^{2}$, this would be equivalent to the power available from the change in gravitational energy per second between Mintaka and earth. The energy would be equivalent to the activity of about $10^{5}$ to $10^{6}$ cortical neurons discharging between 1 and $10 \mathrm{~Hz}$.

Such a population of neurons is not trivial. Typically the average values from the cerebrum during quantitative electroencephalographic (QEEG) activity is about $20 \mu \mathrm{V}$ and when divided by the typical resistivity of extracellular fluid $(\sim 2 \Omega \cdot \mathrm{m})$ and multiplied by the average length of the cerebrum $\left(10^{-1} \mathrm{~m}\right)$, is $10^{-6} \mathrm{~A}$ or a microAmp. Because the unit charge is $1.6 \cdot 10^{-19} \mathrm{~A}$.s per $\mathrm{q}$ (charge), there would be about $10^{13}$ charges per second associated with the cerebral activity. Both the resting membrane potential [1] and action potential are associated with the presence or movement of about $10^{6}$ to $10^{7}$ charges per cell, respectively. This means about $10^{6}$ cortical neurons would be involved.

The alternative approach involves the force density within the entire earth. The gravitational force between Mintaka and the earth $\left(7.52 \cdot 10^{8} \mathrm{~N}\right)$ divided by its volume $\left(1.29 \cdot 10^{21} \mathrm{~m}^{3}\right)$ is $5.83 \cdot 10^{-13} \mathrm{~J} \cdot \mathrm{m}^{-3}$. Within the volume of the typical human cerebrum $\left(1.3 \cdot 10^{-3} \mathrm{~m}^{3}\right)$, the energy is $7.58 \cdot 10^{-16} \mathrm{~J}$. Because the cross sectional area of the cerebrum is involved, the value is $7.58 \cdot 10^{-14} \mathrm{~J} \cdot \mathrm{m}^{-2}$. Multiplication by frequency results in radiant flux density. If we assumed the " $40 \mathrm{~Hz}$ " electroencephalographic activity that is associated with recursive 20 to $25 \mathrm{~ms}$ coherent waves of electromagnetic fields that move in a rostral to caudal direction and are the primary correlates of "human consciousness" $[15,16]$, the power density from the gravitational force between Mintaka and the earth within the human brain would be about $3.03 \cdot 10^{-12} \mathrm{~W} \cdot \mathrm{m}^{-2}$, well within the value of $0.3 \cdot 10^{-11} \mathrm{~W} \cdot \mathrm{m}^{-2}$ of photon flux density associated with imagination in experimental studies.

It is surprising that the astrobiological consequences of gravitational energies within biological masses have not been pursued with more persistence. Given that:

\section{$\mathrm{J}=\mathrm{G} \cdot \mathrm{kg}^{2} \cdot \mathrm{m}^{-1}(1)$,}

Where J=energy in Joules, $\mathrm{kg}$ is the mass of the brain and $\mathrm{m}$ is its length, the gravitational energy associated with the fluctuation in $G$ [17] of about $6.67 \cdot 10^{-14} \mathrm{~m}^{3} \cdot \mathrm{kg}^{-1} \cdot \mathrm{s}^{-2}$ with the average brain mass of 1.5 $\mathrm{kg}$ at a length of $10^{-1} \mathrm{~m}$ is $1.5 \cdot 10^{-12} \mathrm{~J}$ per implicit second. If divided by the total area of the entire cerebral cortices (about two-thirds of which is buried within sulci) according to current "best" measures, $1.8 \cdot 10^{-1}$ $\mathrm{m}^{2}$ [18], the flux density would be $0.8 \cdot 10^{-11} \mathrm{~W} \cdot \mathrm{m}^{-2}$. To pursue this relationship the causal factors, other than instrumental noise, for the lower limit of the stable metrics for $\mathrm{G}$ must still be discerned.

\section{Potential Role of the 5.7 Day Gravitational Variation of the Orbital Stars Distances}

Calculations based upon a stable average distance between earth and Mintaka could be argued to be relatively static and hence of minimal dynamic consequence. What is required is the change in gravitational force associated with one star within the double system as it rotates around the other. To obtain values within $10^{-11} \mathrm{~W} \cdot \mathrm{m}^{-2}$, based upon a variable distance between the stars allegedly responsible for the 5.7 day periodicity, the separation should be about $10^{13} \mathrm{~m}$ or approximately the width of the solar system if Pluto was still considered an integral component. Some references estimate the 
Citation: Dotta BT, Caswell JM, Pesinger MA (2014) Congruence of Coherence Peaks of Daily Geomagnetic Activity and Total Earthquakes with Mintaka's (Orion's Belt) 5.7 Day Periodicity: Potential Implications for Astrobiology. JAstrobiolOutreach 2: 120. doi: $10.41721 / 2332-2519.1000120$

Page 4 of 4

distance between the companion star that rotates around the two proximal bodies to be $1.74 \cdot 10^{13} \mathrm{~m}$.

However this distance is only $0.15 \cdot 10^{-5}$ of the estimated distance of Mintaka and would involve a separation between the orbital stars to be about 1.9 " or when divided by $2 \pi$ to accommodate geometries, around 0.3 ". This would be too small to be directly discerned easily and would require inference from spectral discrepancies of the primary member of the pair. The conceptual challenge to our assumption is that the orbital velocities for two stars separated by $10^{13} \mathrm{~m}$ to accommodate the 5.7 day period would be $\sim 1.27 \cdot 10^{8} \mathrm{~m} \cdot \mathrm{s}^{-1}$ or $0.4 \mathrm{c}$. This is not consistent with contemporary understanding.

If the assumption is correct, then the difference in gravitational forces for a mass equivalent to 20 suns would be $4.34 \cdot 10^{3} \mathrm{~N}$ $\left(\Delta=1.16 \cdot 10^{-5}\right.$ of the value from the mean distance). If this were spread over the hydrogen line $\left(2 \cdot 1 \cdot 10^{-1} \mathrm{~m}\right)$ and divided by the earth's surface, the energy per area would be $1.78 \cdot 10^{-12} \mathrm{~J} \cdot \mathrm{m}^{-2}$. Minakov et al. [19] showed mathematically that the most likely dynamic interaction between gravitational waves and the earth would occur within the second harmonic of the Schumann resonance, $14 \mathrm{~Hz}$. This is within the range of high alpha activity and low beta activity of the human brain. Its occurrence is associated with imagination and thinking. If this frequency is multiplied by the previous value the radiant flux density is $2.5 \cdot 10^{-11} \mathrm{~W} \cdot \mathrm{m}^{-2}$. This quantity is within the range associated with background fluctuations in photon emissions from the earth and from the right hemispheres of people sitting in very dark settings engaging in imaginative thinking.

\section{Conclusions}

The interesting identity between the orbital period of the "double star" within Mintaka and the coherence between daily geomagnetic activity and daily total numbers of seismic events may be a coincidence. However the multiple solutions that indicate the energetic influence from gravitational forces and their energetic equivalents are within the same range: 1$)$ as background $\left(10^{-11} \mathrm{~W} \cdot \mathrm{m}^{-2}\right)$ photon emissions from the earth which increase several days before seismic events, and, 2) that are associated with photon emissions from the human cerebrum during specific cognitive processes, reduce the probability of coincidence. The shared timing does not prove causality. The congruence of quantitative solutions suggests but does not prove that there may be a shared source of variance within this section of the Milky Way that affects the intrinsic periodicity of astrophysical systems and the biological processes they may support.

\section{References}

1. Persinger MA (2010) $10^{-20}$ Joules as a neuromolecular quantum in medicinal chemistry: an alternative approach to myriad molecular pathways? Curr Med Chem 17: 3094-3098.

2. Persinger MA (2014) Terrestrial and lunar gravitational forces upon the mass of a cell: relevance to cell function. International Letters of Chemistry, Physics and Astronomy 2: 15-24.
3. Moraes TA, Barlow PW, Klingelé E, Gallep CM (2012) Spontaneous ultra-weak light emissions from wheat seedlings are rhythmic and synchronized with the time profile of the local gravimetric tide. Naturwissenschaften 99: 465-472.

4. Persinger MA (2014) A possible explanation for the vacuum catastrophe. International Journal of Astronomy and Astrophysics. 4: 178-180.

5. Persinger MA, Lafreniere GF, Dotta BT (2012) Marked increases in background photon emissions in Sudbury, Ontario more than one week before the magnitude $>8.0$ earthquakes in Japan and Chile. International Journal of Geosciences 3: 627-629.

6. Dotta BT, Saroka KS, Persinger MA (2012) Increased photon emission from the head while imaging light in the dark is correlated with changes in electroencephalographic power: support for Bokkon's biophoton hypothesis. Neuroscience Letters 513: 151-154.

7. Li CY, Poo MM, Dan Y (2009) Burst spiking of a single cortical neuron modifies global brain state. Science 324: 643-646.

8. Houweling AR, Brecht M (2008) Behavioural report of single neuron stimulation in somatosensory cortex. Nature 451: 65-68.

9. Vares DAE, Persinger MA (2013) The $~ 3.6$ to $3.7 \mathrm{M}$ paucity in global earthquake frequency: potential coupling to zero point fluctuation force and quantum energies. International Journal of Geosciences 4: 1321-1325.

10. Persinger MA, Koren SA, Lafreniere GF (2008) A neuroquantologic approach to how human thought might affect the universe. NeuroQuantology 6: 262-271.

11. Persinger MA (2014) Quantitative convergence between physicalchemical constants of the proton and the properties of water: implications for sequestered magnetic fields and a universal quantity. International Letters of Chemistry, Physics and Astronomy 2: 1-10.

12. Vares DAE, Persinger MA (2013) Predicting random events from background photon density two days previously: implications for virtualto-matter determinism and changing the future. Journal of NonLocality 2: December ISSN: 2167-6283.

13. Vlaimirsky BM, Bruns AV (1998) Influence of the sector structure of the interplanetary magnetic field on the results of measurements of the Gravitational Constant. Biophysics 43: 720-725.

14. Quinn T, Parks, Speake C, Davis R (2013) Improved determination of g using two methods. Phys Rev Lett 111: 101102.

15. Llinás RR, Paré D (1991) Of dreaming and wakefulness. Neuroscience 44: 521-535.

16. Llinás R, Ribary U (1993) Coherent 40-Hz oscillation characterizes dream state in humans.

17. Persinger MA, St-Pierre LS (2014) Is there a geomagnetic component involved with the determination of G? International Journal of Geosciences 5: 450-452.

18. Pakkenberg B, Gundersen HJ (1997) Neocortical neuron number in humans: effect of sex and age. J Comp Neurol 384: 312-320.

19. Minakov AA, Nikolaenko AP, Rabinovich LM (1992) Gravitational-toelectromagnetic wave convergence in electrostatic field of earthionosphere resonator. Radiophysics and Quantum Electronics 33: 318-323. 